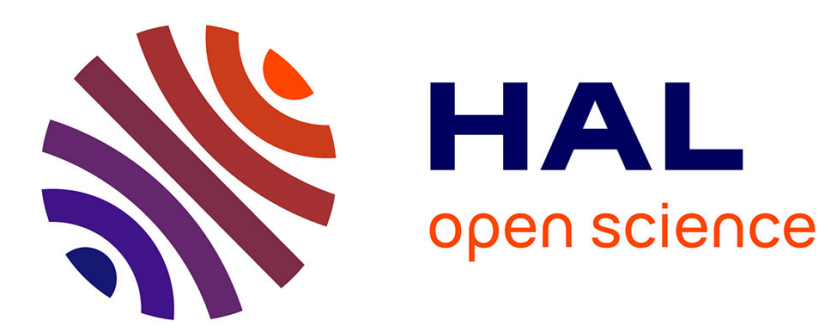

\title{
A remark on the stabilization of partially linear composite systems
}

Abdelhak Ferfera, Abderrahman Iggidr

\section{To cite this version:}

Abdelhak Ferfera, Abderrahman Iggidr. A remark on the stabilization of partially linear composite systems. IEEE Transactions on Automatic Control, 1997, 42 (3), pp.411-414. 10.1109/9.557587 . hal-01860802

\section{HAL Id: hal-01860802 \\ https://hal.science/hal-01860802}

Submitted on 10 Sep 2018

HAL is a multi-disciplinary open access archive for the deposit and dissemination of scientific research documents, whether they are published or not. The documents may come from teaching and research institutions in France or abroad, or from public or private research centers.
L'archive ouverte pluridisciplinaire HAL, est destinée au dépôt et à la diffusion de documents scientifiques de niveau recherche, publiés ou non, émanant des établissements d'enseignement et de recherche français ou étrangers, des laboratoires publics ou privés. 


\section{A Remark on the Stabilization of Partially Linear Composite Systems}

Abdelhak Ferfera, Abderrahmane Iggidr

\begin{abstract}
In this paper, we study the global stabilization, by means of smooth state feedback, of partially linear composite systems. We show how to compute the stabilizing feedback thanks to a weak Lyapunov function for a nonlinear subsystem instead of a stricte one.
\end{abstract}

Keywords- Nonlinear systems, feedback, global stabilization, Lyapunov function.

\section{INTRODUCTION}

Many recent papers (see [1], [2], [6] and references therein) addressed the problem of The global stabilization, by means of state feedback, of nonlinear control systems of the form:

$$
\left\{\begin{array}{l}
\dot{x}=f(x, y) \\
\dot{y}=A y+B u
\end{array}\right.
$$

where $x \in \mathbb{R}^{n}, y \in \mathbb{R}^{p}, u \in \mathbb{R}^{k}, A \in \mathcal{M}_{p, p}(\mathbb{R}), B \in$ $\mathcal{M}_{p, k}(\mathbb{R})$ and $f$ is a smooth vector field such that:

(h1) The pair $(A, B)$ is stabilizble.

(h2) The equilibrium $x=0$ of $\dot{x}=f(x, 0)$ is globally asymptotically stable (G.A.S).

In [6], the authors assumed that the dependence of $f(x, y)$ on $y$ is of the form:

(h3) $f(x, y)=f(x, 0)+G(x, y) C y$, with $C \in \mathcal{M}_{k, p}(\mathbb{R})$ and that both $C$ and $B$ are of full rank.

They proved that there exist a matrix $K \in \mathcal{M}_{k, p}(\mathbb{R})$ and a symmetric positive definite matrix $P \in \mathcal{M}_{p, p}(\mathbb{R})$ satisfying the following three conditions:

(H1) $P(A+B K)+(A+B K)^{T} P=-Q$, with $Q$ symmetric positive $\left({ }^{T}=\right.$ transpose $)$,

(H2) $\left(Q^{1 / 2}, A+B K\right)$ detectable,

(H3) $B^{T} P=C$,

if and only if the linear subsytem

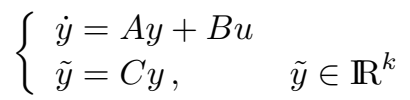

is invertible, weakly minimum phase and with $C B$ symetric positive definite.

Using these conditions, they showed that the system (1) is globally asymptotically stabilizable and they gave the stabilizing feedback:

$$
u(x, y)=K y-\frac{1}{2} G(x, y)^{T} \nabla V(x)
$$

where $V$ is a smooth Lyapunov function satisfying:

$$
\langle\nabla V, f(x, 0)\rangle<0 \forall x \in \mathbb{R}^{n}, x \neq 0
$$

Notice that the existence of such a strict Lyapunov function $V$ is assured by the condition (h1) and the inverse

The authors are with the CONGE Project, INRIA Lorraine \& University of Metz, 4, rue Marconi, 57070 METZ - FRANCE. E-mail: fferfera,iggidr\}@ilm.loria.fr .
Lyapunov theorem (see [3], [5]). Unfortuantly, there is no systematic method to compute a strict Lyapunov function for a given G.A.S system and it is often easier to constructe a weak Lyapunov function for which the hypotheses of LaSalle's invariance principle (see [4]) are satisfied. As an example one can consider the following system which evolves in $\mathbb{R}^{2}$ (Liénard's equation):

$$
\left\{\begin{array}{l}
\dot{x}_{1}=x_{2} \\
\dot{x}_{2}=-g\left(x_{1}\right)-h\left(x_{1}\right) x_{2}
\end{array}\right.
$$

where it is assumed that for all $x \neq 0$ :

$$
x g(x)>0, \quad h(x)>0
$$

and

$$
G(x)=\int_{0}^{x} g(s) d s \rightarrow \infty \text { as }|x| \rightarrow \infty
$$

For this system, it seems difficult to construct a strict Lyapunov function. However LaSalle's theorem can be applied in an obvious way by taking:

$$
V\left(x_{1}, x_{2}\right)=\frac{1}{2} x_{2}^{2}+G\left(x_{1}\right)
$$

In this paper we show that to compute a stabilizing feedback for the system (1), we do not need to have a strict Lyapunov function for:

$$
\dot{x}=f(x, 0)
$$

We also state that the stabilization procedure is still valid when, in the decomposition (h3) of $f$, the matrix $C$ is of rank $m<k$, provided that $C B$ is of full rank.

\section{Notations AND DEFINITIONS}

Before stating the main theorem let us introduce the following notations and definitions.

Definition 1: A $C^{1}$ scalar function $V: \mathbb{R}^{n} \rightarrow \mathbb{R}$ is a weak Lyapunov function for the system on $\mathbb{R}^{n}$ :

$$
\dot{x}=X(x)
$$

if $V$ is positive definite proper and satisfies:

$$
X . V(x) \leq 0, \forall x \in \mathbb{R}^{n}
$$

where $X . V$ is the Lie-derivative of $\mathrm{V}$ along the trajectories of the vector field $X(X . V(x)=\langle\nabla V(x), X(x)\rangle$ where $\langle.,$.$\left.\rangle is the inner product in \mathbb{R}^{n}\right)$.

By a proper function we mean a function $V: \mathbb{R}^{n} \rightarrow \mathbb{R}$ such that $\left\{x \in \mathbb{R}^{n} \mid V(x) \leq \xi\right\}$ is compact for each $\xi>0$. Notice that if the vector field $X$ satisfies the definition 1 then all the trajectories of the system (6) are bounded because of $V$ is proper and its derivative is non positive. For such a vector field, $X_{t}($.$) will denote the flow$ of $X$ defined on $\mathbb{R}^{n}$. A subset $E \in \mathbb{R}^{n}$ is said to be $X$-invariant if for any $x \in E$ on has $X_{t}(x) \in E, \forall t \geq 0$. 
Definition 2: We shall say that the system (6) is of LaSalle-type (L-T) if there exist a weak Lyapunov function $V: \mathbb{R}^{n} \rightarrow \mathbb{R}$ for (6) such that the largest $X$-invariant set contained in $E=\left\{x \in \mathbb{R}^{n} \mid X . V(x)=0\right\}$ is reduced to the origin of $\mathbb{R}^{n}$.

Remark 1: The system (6) is of (L-T) if and only if it is globally asymptotically stable about the origin of $\mathbb{R}^{n}$ (see [4]).

Remark 2: It is often easier to find a function $V$ satisfying the definition 2 than one satisfying (3). This is typically the case for mechanical systems for example.

\section{Stabilization By LASALlE'S INVARIANCE PRINCIPLE}

Theorem 1: Assume that the pair (A,B) is stabilizable and (H1), (H2) and (H3) hold. Then if the system (5) is of L-T, so is the closed-loop system (1) with the (stabilizing) feedback:

$$
u(x, y)=K y-G(x, y)^{T} \nabla V(x)
$$

where $V$ is a weak Lyapunov function for (5) as in the definition 2 .

Proof: First of all, if the linear subsystem (2) satisfies (H1), (H2) and (H3), then it is invertible, weakly minimum phase and with $C B$ symetric positive definite, and it is possible to choose the matrix $K \in \mathcal{M}_{k, p}(\mathbb{R})$ and the symmetric positive definite matrix $P \in \mathcal{M}_{p, p}(\mathbb{R})$ such that:

$$
y^{T} Q y=0 \Rightarrow C y=0
$$

Indeed, as done in [6], one can assume, without loss of generality, that (2) is in the special ccordinate basis (see [7]):

$$
\left\{\begin{array}{l}
\dot{y}_{01}=A_{01} y_{01}+A_{11} y_{1} \\
\dot{y}_{02}=A_{02} y_{02}+A_{12} y_{1} \\
\dot{y}_{1}=D_{01} y_{01}+D_{02} y_{02}+D_{1} y_{1}+C B u \\
\tilde{y}=y_{1}
\end{array}\right.
$$

with $A_{01}$ Hurwitz, $A_{02}+A_{02}^{T}=0$, and take:

$$
K=\left(K_{01}, K_{02}, K_{1}\right)
$$

with:

$$
\begin{aligned}
K_{01} & =-(C B)^{-1} D_{01}+A_{11}^{T} P_{01} \\
K_{02} & =(C B)^{-1} D_{02}+A_{12}^{T} \\
K_{1} & =(C B)^{-1} D_{1}+\frac{1}{2} I
\end{aligned}
$$

and:

$$
P=\left(\begin{array}{ccc}
P_{01} & 0 & 0 \\
0 & I & 0 \\
0 & 0 & (C B)^{-1}
\end{array}\right)
$$

with $P_{01}$ symmetric positive definite such that:

$$
P_{01} A_{01}+A_{01}^{T} P_{01}=-I
$$

This particular choice of $K$ and $P$ leads to:

$$
Q=\left(\begin{array}{lll}
I & 0 & 0 \\
0 & 0 & 0 \\
0 & 0 & I
\end{array}\right)
$$

Hence, $y^{T} Q y=\left\|y_{01}\right\|^{2}+\left\|y_{1}\right\|^{2}$ and so:

$$
y^{T} Q y=0 \Rightarrow C y=y_{1}=0
$$

Assume now that the system (5) is of L-T, and set $X(x)=f(x, 0), x \in \mathbb{R}^{n}$. Let $V$ be a weak Lyapunov function for (5) as in the definition 2 and denote by $\Omega$ the largest invariant set by $X$ contained in the locus $E=$ $\left\{x \in \mathbb{R}^{n} \mid X . V(x)=0\right\}$. By hypotheses $\Omega=\{0\}$. From (H1) - (H3), for $x \in \mathbb{R}^{n}$ and $y \in \mathbb{R}^{p}$, set:

$$
Z(x, y)=\left(\begin{array}{l}
f(x, y) \\
h(x, y)
\end{array}\right)
$$

where:

$$
\begin{aligned}
h(x, y) & =A y+B u(x, y \\
& =(A+B K) y-B G(x, y)^{T} \nabla V(x)
\end{aligned}
$$

and define (see [6]):

$$
W(x, y)=V(x)+\frac{1}{2} y^{T} P y
$$

$W$ is of class $C^{1}$, definite positive and proper, and its derivative along the trajectories of the vector field $Z$ is given by:

$$
\begin{aligned}
\dot{W}(x, y)= & Z . W(x, y) \\
= & \langle Z(x, y), \nabla W(x, y)\rangle \\
= & X \cdot V(x)+\langle\nabla V(x), G(x, y) C y\rangle \\
& +\frac{1}{2} y^{T} Q y+\left\langle y,-P B G(x, y)^{T} \nabla V(x)\right\rangle \\
= & X . V(x)+\frac{1}{2} y^{T} Q y \\
& +\left\langle y, C^{T} G(x, y)^{T} \nabla V(x)\right. \\
& \left.-P B G(x, y)^{T} \nabla V(x)\right\rangle
\end{aligned}
$$

So, by use of (H3) one has:

$$
\dot{W}(x, y)=X . V(x)+\frac{1}{2} y^{T} Q y \leq 0
$$

Notice that all the trajectories of the closed-loop system are bounded because of $W$ is proper and its derivative is non positive. Set:

$$
\begin{aligned}
\tilde{E} & =\left\{(x, y) \in \mathbb{R}^{n+p} \mid Z \cdot W(x, y)=0\right\} \\
& =\left\{(x, y) \in \mathbb{R}^{n+p} \mid X \cdot V(x)=0, \text { and } y^{T} Q y=0\right\}
\end{aligned}
$$

According to LaSalle's theorem (see [4] pp 66-67) all the solutions of the closed-loop system tend to $\tilde{\Omega}$ the largest invariant set by $Z$ contained in $\tilde{E}$. in order to prove the theorem 1 let us show that $\tilde{\Omega}$ is the origin of $\mathbb{R}^{n+p}$. By (8), on $\tilde{E}$ the vector field $Z$ is given by:

$$
Z(x, y)=\left(\begin{array}{l}
X(x) \\
Y(x, y)
\end{array}\right)
$$


where:

$$
\begin{aligned}
X(x) & =f(x, 0) \\
Y(x, y) & =(A+B K) y-B G(x, y)^{T} \nabla V(x)
\end{aligned}
$$

so that on $\tilde{E}$ the closed-loop system becomes:

$$
\left\{\begin{array}{l}
\dot{x}=f(x, 0)=X(x) \\
\dot{y}=(A+B K) y-B G(x, y)^{T} \nabla V(x)
\end{array}\right.
$$

Let $(x(t), y(t))$ be a solution of the above system with $(x(0), y(0))=(x, y) \in \tilde{\Omega}$. Since $\tilde{\Omega}$ is $Z$-invariant we have $(x(t), y(t)) \in \tilde{\Omega}$ for all $t \geq 0$. But one has:

$$
\frac{d}{d t}(x(t))=X(x(t))
$$

so that $x(t)=X_{t}(x)$. Consider now the following set:

$$
M=\left\{x \in \mathbb{R}^{n} \mid \exists y \in \mathbb{R}^{p} \text {, such that }(x, y) \in \tilde{\Omega}\right\}
$$

If $x \in M$ then $(x, y) \in \tilde{\Omega}$ for some $y \in \mathbb{R}^{p}$, and for all $t \geq 0$, $(x(t), y(t))=\left(X_{t}(x), y(t)\right) \in \tilde{\Omega}$ since $\tilde{\Omega}$ is $Z$-invariant, that implies $X_{t}(x) \in M$. Then $M$ is $X$-invariant wich implies that $M \subset \Omega=\{0\}$. So we have shown that:

$$
(x, y) \in \tilde{\Omega} \Rightarrow x=0
$$

Since $x(t)=0$ for all $t \geq 0, y(t)$ becomes a solution of $\dot{y}=(A+B K) y$ and $y(t)^{T} Q y(t)=0$ for all $t \geq 0$. Hence, from (H2) one deduce that $y(t)=0$ for all $t \geq 0$ and so $\tilde{\Omega}=\{(0,0)\}$ which completes the proof of theorem 1 .

Example: Consider the following system evolving in $\mathbb{R}^{4}$ :

$$
\left\{\begin{array}{l}
\dot{x}_{1}=x_{2}+\left(x_{1} y_{1}\right)^{4 / 3} y_{2} \\
\dot{x}_{2}=-x_{1}^{5 / 3}-x_{1}^{4 / 3} x_{2}+\left(x_{1} y_{2}\right)^{4 / 3} y_{2} \\
\dot{y}_{1}=y_{2} \\
\dot{y}_{2}=u
\end{array}\right.
$$

The subsystem:

$$
\left\{\begin{array}{l}
\dot{x}_{1}=x_{2} \\
\dot{x}_{2}=-x_{1}^{5 / 3}-x_{1}^{4 / 3} x_{2}
\end{array}\right.
$$

is of the form (4) and so it is of L-T thanks to the weak Lyapunov function:

$$
V\left(x_{1}, x_{2}\right)=\frac{1}{2} x_{2}^{2}+\frac{3}{8} x_{1}^{8 / 3}
$$

Besides, the assumptions $\mathbf{H 1}$ - H3 hold for the linear subsystem:

$$
\left\{\begin{array}{l}
\dot{y}_{1}=y_{2} \\
\dot{y}_{2}=u \\
\tilde{y}=y_{2}
\end{array}\right.
$$

with $K=\left(-1,-\frac{1}{2}\right)$ and $P=I$. Hence the system (9) satisfies the conditions of the theorem 1 and so it is stabilizable thanks to the feedback:

$$
u\left(x_{1}, x_{2}, y_{1}, y_{2}\right)=-y_{1}-\frac{1}{2} y_{2}-x_{1}^{3} y_{1}^{4 / 3}-x_{2}\left(x_{1} y_{2}\right)^{4 / 3}
$$

Remark 3: Throughout all this work it is supposed that in (h3) one has $C \in \mathcal{M}_{k, p}(\mathbb{R})$, so that the linear subsytem (2) has the same number of inputs and outputs. This restriction can be relaxed by assuming that $C \in \mathcal{M}_{m, p}(\mathbb{R})$, $m \leq k$, and (2) is right invertible, weakly minimum phase and with $C B$ of full rank. To make this, notice that, as mentionned in [6], if $m=k$ the assumption $C B$ symetric positive definite can be replaced by $C B$ nonsingular thanks to the use of a static precompensator $u=(C B)^{-1} \tilde{u}$. Then the remark is deduced from the following proposition.

Proposition 1: Assume that $m<k \leq p$ and that both $B$ and $C B$ are of full rank. Then there exists a matrix function $G^{\prime}(x, y) \in \mathcal{M}_{n, k}(\mathbb{R}), x \in \mathbb{R}^{n}, y \in \mathbb{R}^{p}$, and a constant matrix $C^{\prime} \in \mathcal{M}_{k, p}(\mathbb{R})$ such that $C^{\prime} B$ is nonsingular and:

$$
G(x, y) C=G^{\prime}(x, y) C^{\prime}, \quad \forall(x, y) \in \mathbb{R}^{n+p}
$$

Furthermore, if (2) is weakly minimum phase, so is the linear system:

$$
\left\{\begin{array}{l}
\dot{y}=A y+B u \\
\tilde{y}^{\prime}=C^{\prime} y, \quad \tilde{y}^{\prime} \in \mathbb{R}^{k}
\end{array}\right.
$$

Proof: From the full rank property of $B$ and $C B$, it is always possible to choose $\tilde{C} \in \mathcal{M}_{k-m, p}(\mathbb{R})$ in such a way that the block-matrix:

$$
C^{\prime}=\left(\begin{array}{l}
C \\
\tilde{C}
\end{array}\right)
$$

satisfies $C^{\prime} B$ nonsingular. For such a choice, and taking $G^{\prime}(x, y)=(G(x, y), 0)$, one has:

$$
G(x, y) C=G^{\prime}(x, y) C^{\prime}
$$

Furthermore, from (11) one can deduce that the zero dynamics of (10) are included in those of (2) which completes the proof of the proposition.

\section{REFERENCES}

[1] P.V. Kokotovic and H.J. Sussmann. A positive real condition for global stabilization of nonlinear systems. Systems \& Control Letters, (13):125-133, (1989).

[2] P.V. Kokotovic and H.J. Sussmann. The peaking Phenomenon and the global Stabilization of nonlinear systems. I.E.E.E Trans. Automat. Control, Vol.36, No 4:424-440, April 1991.

[3] J. Kurzweil. On the inversion of liapunov's second theorem on stability of motion. AMS Translations, 24:19-77, (1963).

[4] J. LaSalle and S. Lefschetz. Stability by Liapunov's direct method with applications. Academic Press, New-York, (1961).

[5] J.L. Massera. Contribution to stability theory. Annals of Mathematics, (64):182-206, (1956).

[6] A. Saberi, P.V. Kokotovic and H.J. Sussmann. Global stabilization of partially linear composed systems. SIAM J. Control and Optimisation, (28):1491-1503, (1990).

[7] P. Sannuti and A. Saberi. Special coordinates basis for multivariable linear systems - finite and infinite zero structure, squaring down and decoupling. Int. J. Control, (45):1655-1704, (1987). 